\title{
Development of a Refined Rollover Model That Recognizes the Effects of Suspension and Tire Deformation
}

\author{
Xiaowen Song, Jie Li, and Aiqun Xu \\ Department of Mechanical Engineering, Zhejiang University, Hangzhou, Zhejiang 310027, China \\ Correspondence should be addressed to Xiaowen Song; songxw@zju.edu.cn
}

Received 13 November 2012; Accepted 15 December 2012

Academic Editor: Tsung-Chih Lin

Copyright (c) 2013 Xiaowen Song et al. This is an open access article distributed under the Creative Commons Attribution License, which permits unrestricted use, distribution, and reproduction in any medium, provided the original work is properly cited.

\begin{abstract}
Vehicle rollover represents one of the most dangerous traffic accidents in the world. To improve the antirollover capability of a vehicle, we established an improved rollover model with a particular focus on the effects of independent suspensions and the lateral deformation of the tire. Based on this model, we further developed a new method to mitigate the rollover occurrence by adjusting the stiffness of the spring and the damping coefficient of the damper. Through simulation tests with a brand of SUV, we demonstrated that these adjustments improved the mitigation control as evidenced by better confined steady value and decreased overshoot of the roll angle.
\end{abstract}

\section{Introduction}

Rollover incidents are severe safety problems that occur in all classes of vehicles. According to the USA National Highway Traffic Safety Administration's rollover research program [1], rollovers account for $8 \%$ of total traffic accidents, and, notably, rollover-related severe and fatal injuries are as high as $31 \%$ and $21 \%$, respectively. Vehicle rollover can be caused by multiple factors, including vehicle parameters, excessive steering, and soft terrain. Thus, studies aiming to enhance vehicle rollover resistance have been extensively pursued in recent years by numerous researchers from academic institutions as well as automobile industries. The reported studies can be classified into two categories: passive safety and active safety. The former one includes two aspects: (1) configuration of vehicle parameters that are sensitive to the static stability factor (SSF) and (2) detection of rollover signs in advance to signal the vehicle operators to take necessary reactions. Hac proposed a simple and insightful model to predict the propensity of vehicle rollover and recommended a few guidelines for passive suspension design [2]. Bouton et al. designed a rollover risk indicator specifically for offroad vehicles by incorporating the environmental properties and grip conditions into the design [3]. Yi et al. described a new methodology to design model-based estimators to detect possible rollovers. Moreover, the proposed estimator can give useful estimates on the roll angle and roll rate, two critical measurements for the risk of vehicle rollover [4]. Seongjin Yim designed a preview controller with the help of a GPS or an inertial measurement unit which can predict a driver's steering input [5]. Mathieu et al. developed an algorithm to predict the rollover risk for all-terrain vehicle by means of an on-line estimation of a stability criterion [6]. In addition to the previous passive safety control, there are also four kinds of active safety control techniques, namely, four-wheel steering, active suspension, antiroll bar, and differential braking, which are independent of the operators' routine handling and are triggered only in response to emergency conditions. Chen and Peng derived an antirollover control algorithm based on time-to-rollover (TTR) metric and combined with human-in-the-loop evaluations [7]. Lu et al. developed a two-layer hierarchical strategy for global integrated control of chassis subsystems associated with controlled suspension, braking system, and steering system [8]. Solmaz et al. utilized differential-braking-based rollover controllers to keep the values of vehicle outputs below certain level, and by doing so, the controllers were shown to be stable under varied vehicle speeds [9]. Cherian et al. described a methodology 
aiming to apply model-based design to the development and optimization of the vehicle stability control systems using CarSim and Simulink [10]. Vasilio Tsourapas et al. proposed a new method of identifying real-time predictive lateral load transfer ratio for rollover prevention [11]. Hajishafieiha Peiman proposed an active roll control system with a fuzzy logic controller $[12,13]$.

Since light-duty vehicles are featured with relatively high center of mass and considerable deformations of suspensions and tires, the SSF of light-duty vehicles should be significantly reduced by $25 \%$ according to Alexksander $\mathrm{Hac}$ [2]. In this paper, we established a vehicle rollover model dealing with the changes of suspensions and tires, which has never been reported before. Using this model, we further studied the transient and steady performance of a vehicle with input of lateral acceleration and first uncovered that an optimal relationship between spring stiffness and damper damping coefficient is critical for a proper vehicle dynamic response. With this finding, we then developed a control logic to optimize this relationship in order to limit the vehicle roll state in both transient and steady phases. The effect of the proposed control logic is demonstrated by a set of simulation tests carried out according to the Fishhook la test regulations recommended by NHTSA [14].

\section{Rollover Model}

Following the traditional approach, we utilize the simplified three-freedom vehicle model as depicted in Figure 1.

In Figure 1(a), $x$ - $y$ plane, the lateral forces over the front and rear tires are denoted by $F_{y 1}$ and $F_{y 2}$. The sideslip angle $\beta$ and the roll angle $\phi$ are assumed to be minor. The total forces and torques over the whole vehicle are given by

$$
\begin{gathered}
\sum F_{y}=F_{y 1} \cos \delta+F_{y 2}=m a_{y} \\
\sum M_{z}=a F_{y 1} \cos \delta-b F_{y 2}=I_{z} \dot{\omega}_{r}
\end{gathered}
$$

where $m$ is the total mass of the vehicle, the lengths $a$ and $b$ are defined as shown in Figure 1(a), $a_{y}$ is the lateral acceleration, $\dot{\omega}_{r}$ is the yaw acceleration, and $I_{z}$ is the yaw moment of the vehicle inertia.

In addition, the lateral forces $F_{y 1}=k_{1} \alpha_{1}, F_{y 2}=k_{2} \alpha_{2}$, where $k_{1}$ and $k_{2}$ are the cornering stiffness of front and rear tires, respectively, $\alpha_{1}$ and $\alpha_{2}$ are the slip angles of front and rear tires, $\alpha_{1}=\beta+a \omega_{r} / v_{x}-\delta, \alpha_{2}=\beta-b \omega_{r} / v_{x}, v_{x}$ is the longitudinal velocity, and $\omega_{r}$ is the yaw rate. Consequently, the following equation is derived:

$$
\begin{gathered}
a_{y}=\frac{\left(k_{1}+k_{2}\right) \beta}{m}+\frac{\left(a k_{1}-b k_{2}\right) \omega_{r}}{m v_{x}}-\frac{k_{1} \delta}{m}, \\
\dot{\omega}_{r}=\frac{\left(a k_{1}-b k_{2}\right) \beta}{I_{z}}+\frac{\left(a^{2} k_{1}+b^{2} k_{2}\right) \omega_{r}}{I_{z} v_{x}}-\frac{a k_{1} \delta}{I_{z}} .
\end{gathered}
$$

In Figure 1(b), $y$ - $z$ plane, the total forces and torques over the whole vehicle are given by

$$
\begin{gathered}
\sum F_{z}=F_{n 1}+F_{n 2}-m g=0, \\
\sum M_{x}=m g h \sin \phi+m a_{y}\left(h_{0}+\Delta h-h\right) \\
+F_{n 2}\left(\frac{T}{2}+\Delta T\right)-F_{n 1}\left(\frac{T}{2}-\Delta T\right)=I_{x} \ddot{\phi},
\end{gathered}
$$

where $\ddot{\phi}$ is the body roll acceleration, $h_{0}$ is the height of the vehicle's center of gravity (CG) standing above the ground level, $h$ is the distance between the vehicle CG and the assumed roll axis, $T$ is the width of the vehicle track, and $\Delta h$ and $\Delta T$ are the deformations of suspensions and tires. $F_{n 1}$ and $F_{n 2}$ are normal forces over the left and right wheels, and $I_{x}$ is the roll moment of vehicle inertia.

The restoring force $F_{a}$ in Figure 1(c) is associated with the roll movement, $F_{a}=\left(k_{\phi} \phi+c_{\phi} \dot{\phi}\right) / h$, where $k_{\phi}$ and $c_{\phi}$ are the total roll stiffness and roll damping of suspensions and $\dot{\phi}$ is the roll rate.

The total torques over the body with respect to the roll axis are given by

$$
\sum M_{x s}=m_{s} a_{y} h+m_{s} g h \sin \phi-F_{a} h=I_{x s} \ddot{\phi}
$$

where $m_{s}$ is the sprung mass that represents the total vehicle mass $m$ excluding the suspension and tire and $I_{x s}$ is the roll moment for the inertia of the sprung mass.

Compared with the sprung mass, the unsprung mass is much smaller. Therefore, we can suppose that $m_{s}=m$ and $I_{x s}=I_{x}$, and establish (5) as follows:

$$
\begin{gathered}
\ddot{\phi}=\frac{m h\left(a_{y}+g \sin \phi\right)}{I_{x}}-\frac{k_{\phi} \phi}{I_{x}}-\frac{c_{\phi} \dot{\phi}}{I_{x}}, \\
F_{n 1}-F_{n 2}=\frac{2 m a_{y}\left(h_{0}+\Delta h-2 h\right)}{T}+\frac{2 m g \Delta T}{T} \\
-\frac{2\left(k_{\phi} \phi+c_{\phi} \dot{\phi}\right)}{T}, \\
F_{n 1}+F_{n 2}=m g, \\
\operatorname{LTR}=\left(\frac{F_{n 1}-F_{n 2}}{F_{n 1}+F_{n 2}}\right),
\end{gathered}
$$

where LTR stands for the load transfer ratio, an important criteria to evaluate the vehicle status [15].

Since $a_{y}=\dot{v}_{y}+v_{x} \omega_{r}, \beta=v_{y} / v_{x}$, after introducing the state vector $x=\left[\begin{array}{llll}v_{y} & \omega_{r} & \dot{\phi} & \phi\end{array}\right]^{T}$, the motions of this model can be described by

$$
\dot{x}=A x+B \delta,
$$




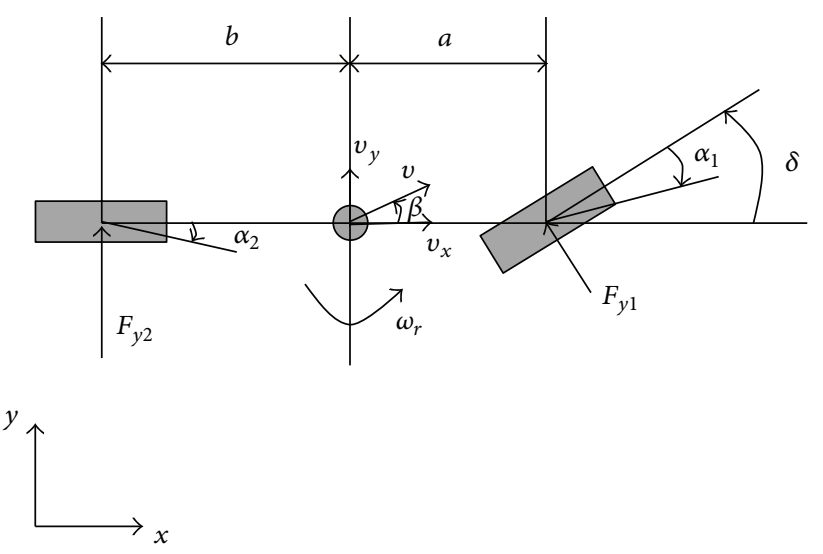

(a) $x-y$ of the simplified model

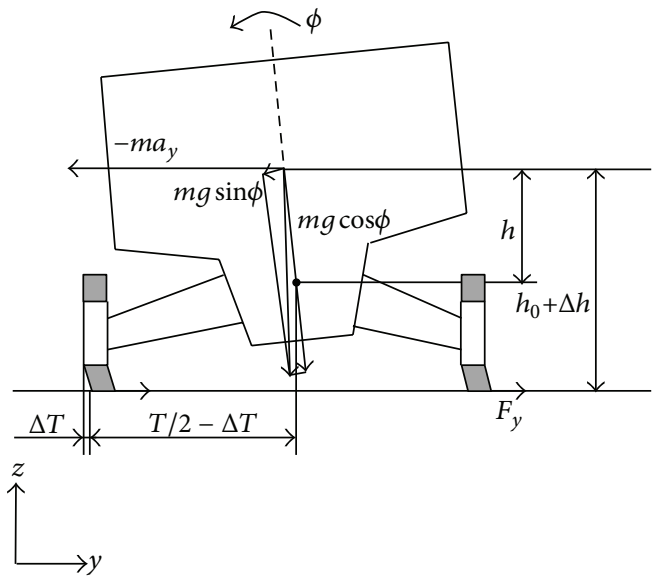

(b) $y-z$ of the simplified model

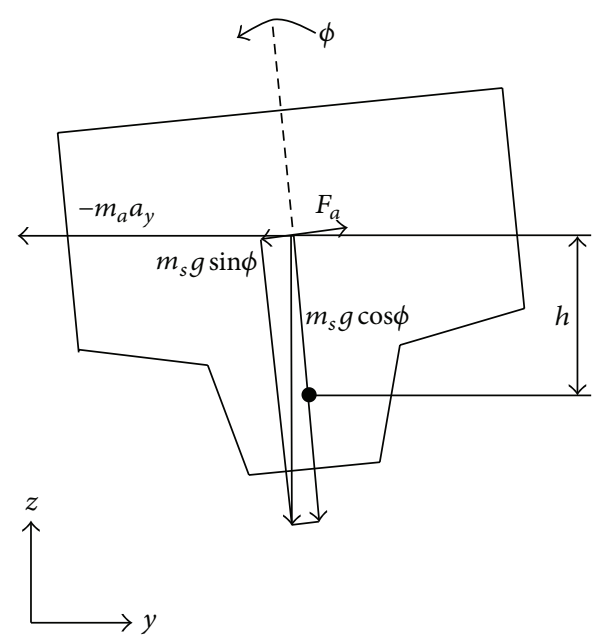

(c) the vehicle body model

FIGURE 1: Simplified vehicle rollover model.

where

$$
\begin{gathered}
A=\left[\begin{array}{cccc}
\frac{k_{1}+k_{2}}{m v_{x}} & \frac{a k_{1}-b k_{2}}{m v_{x}}-v_{x} & 0 & 0 \\
\frac{a k_{1}-b k_{2}}{I_{z} v_{x}} & \frac{a^{2} k_{1}+b^{2} k_{2}}{I_{z} v_{x}} & 0 & 0 \\
\frac{k_{1}+k_{2}}{I_{x} v_{x}} & \frac{a k_{1}-b k_{2}}{I_{z} v_{x}} & -\frac{c_{\phi}}{I_{x}} & \frac{m g h-k_{\phi}}{I_{x}} \\
0 & 0 & 1 & 0
\end{array}\right], \\
B=\left[\begin{array}{ccc}
-\frac{k_{1}}{m}-\frac{a k_{1}}{I_{z}} & -\frac{k_{1}}{I_{x}} & 0
\end{array}\right]^{T}
\end{gathered}
$$

\section{Mitigation Control}

The running vehicle intends to overshoot in its response to a suddenly applied lateral acceleration. Therefore, the inner wheels may have lifted off during the steering when the applied acceleration is lower than the nominal permit value that is in compliance with the steady-state laws [16].
From (5), we can see

$$
\ddot{\phi}=\frac{m h\left(a_{y}+g \sin \phi\right)}{I_{x}}-\frac{k_{\phi} \phi}{I_{x}}-\frac{c_{\phi} \dot{\phi}}{I_{x}},
$$

where the roll angle $\phi$ is small enough to get $\sin \phi \approx \phi$, and after applying Laplace law to the aforementioned differential equation, we established the following new equation:

$$
\frac{\phi(s)}{a_{y}(s)}=\frac{\left(k_{\phi}-m g h\right) / I_{x}}{s^{2}+\left(c_{\phi} / I_{x}\right) s+\left(k_{\phi}-m g h\right) / I_{x}} \cdot \frac{m h / I_{x}}{\left(k_{\phi}-m g h\right) / I_{x}},
$$

where the angular frequency is

$$
\omega_{n}=\left[\frac{\left(k_{\phi}-m g h\right)}{I_{x}}\right]^{1 / 2}
$$




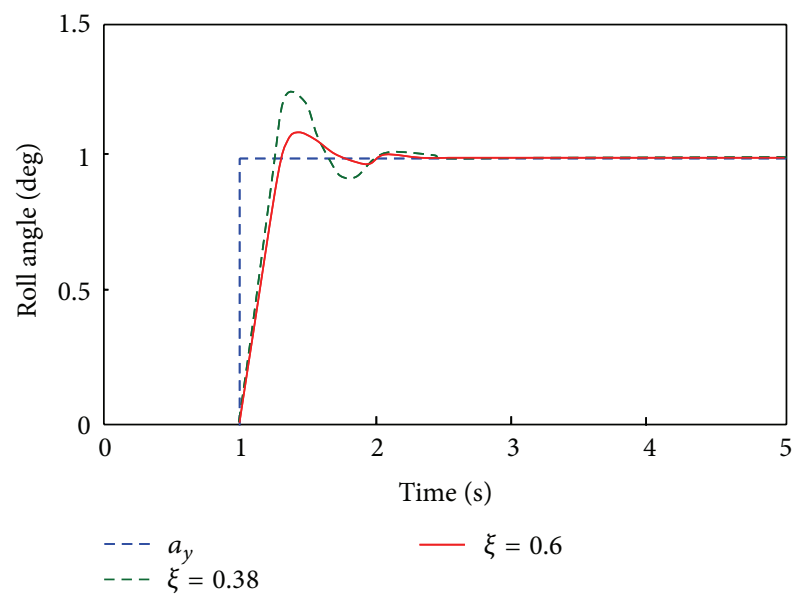

FIgURE 2: $\phi-a_{y}$ transient response.

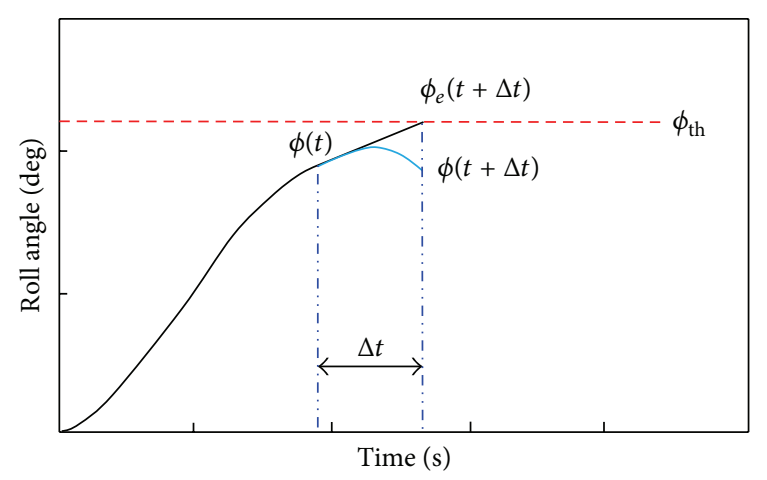

FIgURE 3: The prospective control on the roll angle.

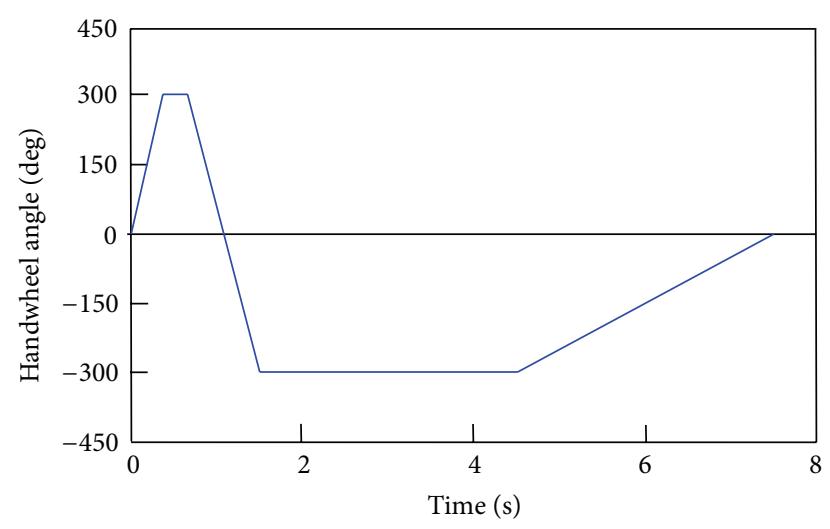

FIgURE 4: Fishhook la maneuver.

and the damping ratio is

$$
\xi=\frac{c_{\phi}}{2\left[\left(k_{\phi}-m g h\right) I_{x}\right]^{1 / 2}} .
$$

When the input $a_{y}$ is set to be the unit step function, the overshoot is

$$
M p=\exp \left(-\frac{\xi \pi}{\left(1-\xi^{2}\right)^{1 / 2}}\right)
$$

TABLE 1: Parameters of the simulated SUV.

\begin{tabular}{lcc}
\hline Parameter & Value & Unit \\
\hline$m$ & 1460 & $(\mathrm{~kg})$ \\
$v_{x}$ & 60 & $(\mathrm{~km} / \mathrm{h})$ \\
$h_{0}$ & 0.628 & $(\mathrm{~m})$ \\
$h$ & 0.35 & $(\mathrm{~m})$ \\
$T$ & 1.68 & $(\mathrm{~m})$ \\
$a$ & 1.233 & $(\mathrm{~m})$ \\
$b$ & 1.327 & $(\mathrm{~m})$ \\
$k_{\phi}$ & 45000 & $(\mathrm{~N} \cdot \mathrm{m} / \mathrm{rad})$ \\
$c_{\phi}$ & 3600 & $(\mathrm{~N} \cdot \mathrm{m} \cdot \mathrm{s} / \mathrm{rad})$ \\
$a_{1}$ & 0.13 & $\mathrm{Null}$ \\
$a_{2}$ & 0.225 & $\mathrm{Null}$ \\
$c_{1}$ & 80200 & $(\mathrm{~N} / \mathrm{rad})$ \\
$c_{2}$ & 160000 & $(\mathrm{~N} / \mathrm{rad})$ \\
$I_{x}$ & 550 & $\left(\mathrm{~kg} \cdot \mathrm{m}^{2}\right)$ \\
$I_{z}$ & 1400 & $\left(\mathrm{~kg} \cdot \mathrm{m}^{2}\right)$ \\
\hline
\end{tabular}

At this point, we found that vehicle dynamics is determined by the first part of (9), whereas the second part defines the vehicle steady state. These findings form the base of the mitigation control referred to in the rest of this section.

A specific brand of midsize SUV was used as the test vehicle in this study, of which the relevant parameters are shown in Table 1.

With the rollover model and vehicle parameters, we achieved the damping ratio of the original vehicle, $\xi=0.38$, and the overshoot value of the roll angle at $36 \%$ as shown in Figure 2. Thus, the damping ratio needs to be raised in order to reduce the overshoot value. In other words, we can improve the restoring force $F_{a}$ by increasing the roll stiffness $k_{\phi}$ or the roll damping $c_{\phi}$. However, it needs to bear in mind that the roll stiffness $k_{\phi}$ and the roll damping $c_{\phi}$ must be increased simultaneously. Otherwise, the damping ratio $\xi$ may be decreased.

When the gain in roll stiffness $\Delta k_{\phi}$ and the gain in roll damping $\Delta c_{\phi}$ are 1500 and 2150, respectively, the damping ratio $\xi$ will be 0.6 , and the overshoot will be much smaller than the original one as shown in Figure 2. As indicated by the calculated values of $\Delta k_{\phi} / k_{\phi}=3.3 \%$ and $\Delta c_{\phi} / c_{\phi}=$ $59.7 \%$, a small gain in the roll stiffness results in substantial changes in roll damping in a disproportional manner. This disproportion will become more severe if the damping ratio is higher. Therefore, it is necessary to establish an adjusting relationship between the roll stiffness and the roll damping to ensure a proper match between them.

In theory, the overshoot is nearby zero if the damping ratio equals one. However, as we know, high roll stiffness and roll damping cost vehicle ride comfort. For a balance purpose, we assigned the compromised damping ratio $\xi$ at a normal factory-set value.

There are many factors that can influence the roll stiffness $k_{\phi}$ and the roll damping $c_{\phi}$, such as springs, dampers, and bushings. Here, we adopted two ratio factors, $a_{1}$ and $a_{2} \cdot a_{1}$ 


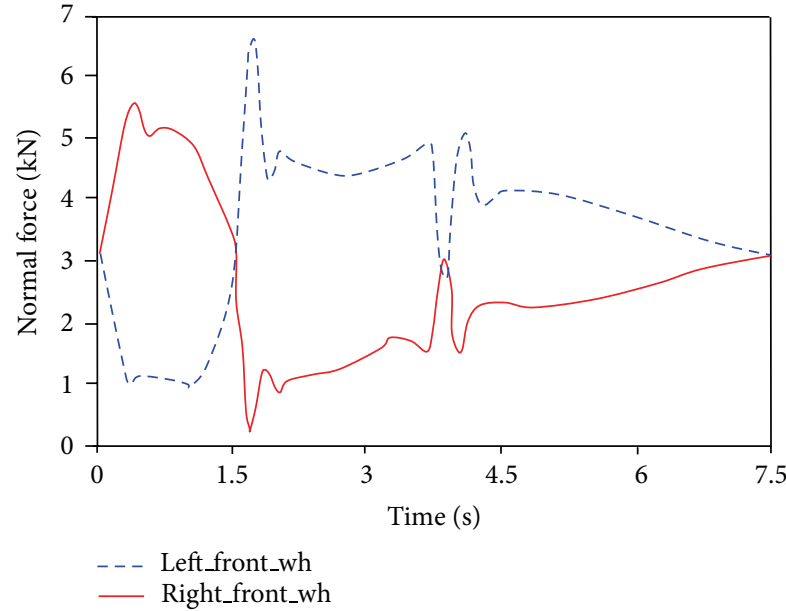

(a)

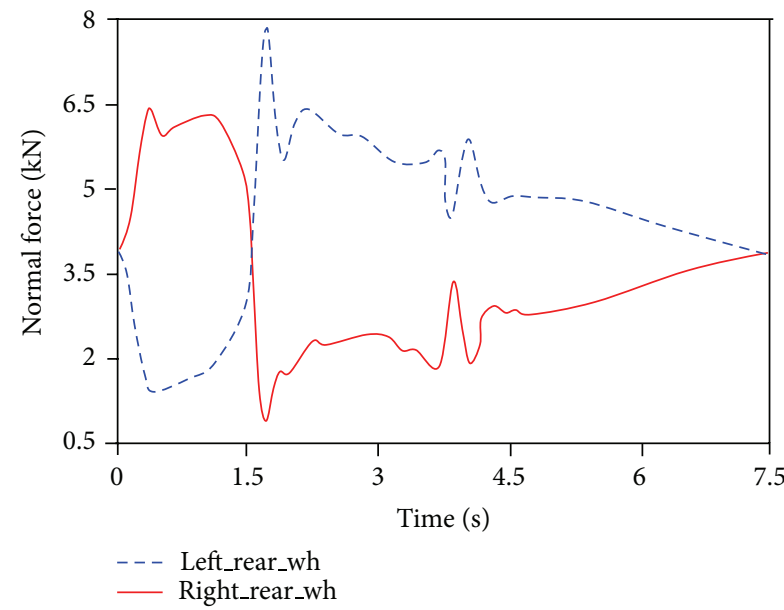

(b)

FIGURE 5: Normal forces on four wheels of the original vehicle.

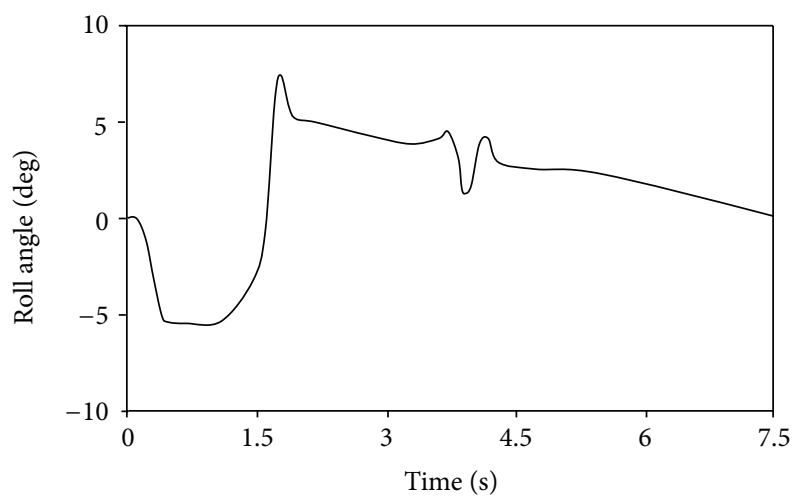

(a)

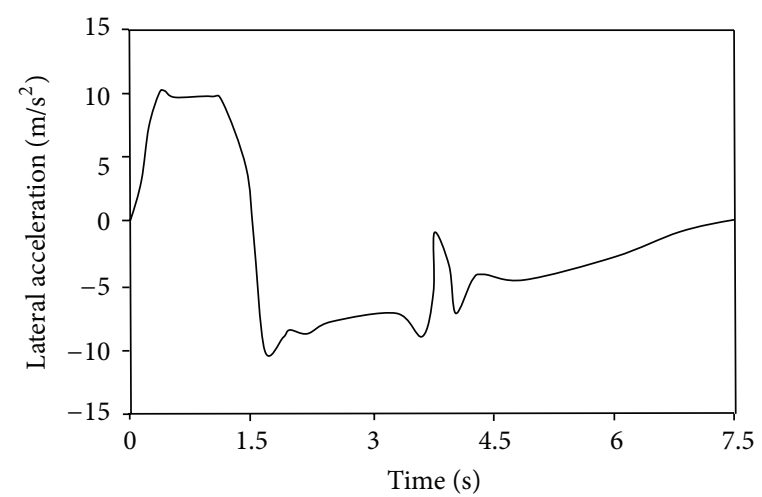

(b)

Figure 6: Roll angle and lateral acceleration of the original one.

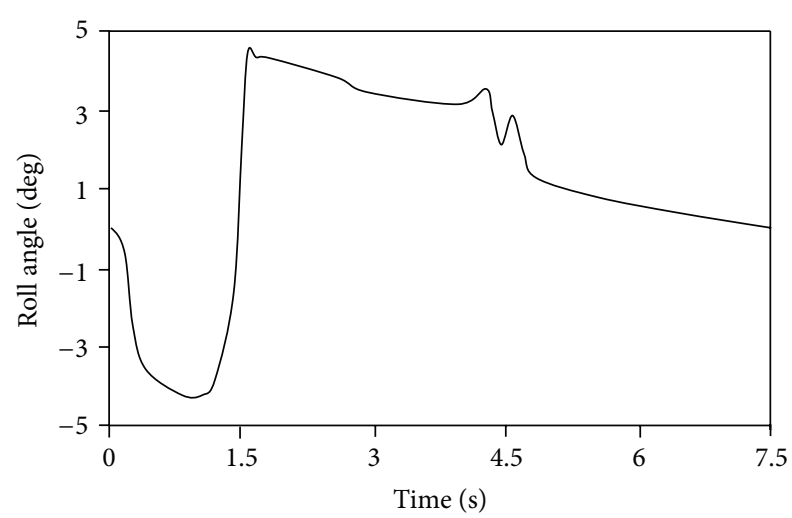

Figure 7: Roll angle of the modified.

couples the roll stiffness $k_{\phi}$ and the spring stiffness $k$ together in the form of $k_{\phi}=a_{1} k$.

Similarly, $a_{2}$ serves a link between the roll damping $c_{\phi}$ and damper damping coefficient $c$ in the form of $c_{\phi}=a_{2} c$.
With the compromised damping ratio value, by substituting the original values of $k_{0}$ and $c_{0}$ and the gains of $\Delta k$ and $\Delta c$ in the spring and the damper into (11), we obtained the following equation:

$$
\begin{aligned}
\Delta k= & \frac{a_{2}^{2}}{1.44 a_{1} I_{x}} \Delta c^{2}+\frac{a_{2}^{2} c_{0}}{0.72 a_{1} I_{x}} \Delta c \\
& +\left(\frac{a_{2}^{2} c_{0}^{2}}{1.44 a_{1} I_{x}}+\frac{m g h}{a_{1}}-k_{0}\right) .
\end{aligned}
$$

We then attempted to identify the value of $\Delta k$ (or $\Delta c$ ) for danger prevention. As mentioned previously, the second part of (9) determines the ultimate roll angle value in the steady state, and the prospective control on the roll angle is shown in Figure 3 , where $\Delta t$ is a time period proceeding to the vehicle rollover, $\phi_{\mathrm{th}}$ is the desired threshold value of the roll angle, and $\phi_{e}(t+\Delta t)$ is the estimated value of the roll angle. If $\phi_{e}(t+$ $\Delta t)>\phi_{\text {th }}$, mitigation control will be activated in the buffer 


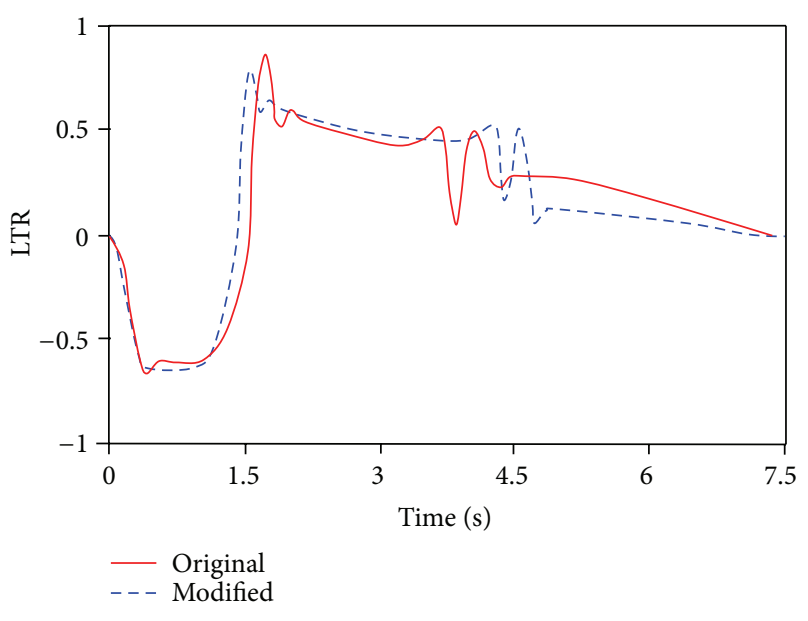

FIGURE 8: LTRs of the two prototypes.

period time of $\Delta t$, and if we keep $\phi(t+\Delta t)$ the same as $\phi(t)$, then

$$
\begin{gathered}
\phi(t)=\phi(t+\Delta t), \\
\phi(t)=\frac{m h}{a_{1} k-m g h} a_{y}(t), \\
\phi(t+\Delta t)=\frac{m h}{a_{1} k^{*}-m g h} a_{y e}(t+\Delta t), \\
\Delta k=k^{*}-k,
\end{gathered}
$$

where $a_{y e}$ is the estimated value of the lateral acceleration. $\Delta t$ is the value in relevance to the system control frequency of a given active suspension.

As deduced from (14), the value of $\Delta k$ is:

$$
\Delta k=\frac{a_{y e}(t+\Delta t)-a_{y}(t)}{a_{1} a_{y}(t)}\left(a_{1} k-m g h\right)
$$

Finally, (13) and (15) on $\{\Delta k, \Delta c\}$ give rise to the mitigation control that stabilizes the vehicles.

\section{Simulation Results}

In order to verify the proposed mitigation control, simulation tests are conducted according to NHTSA's Fishhook la test description, and the steering maneuver patterns are shown in Figure 4.

Using the vehicle parameters in Table 1 and the multibody simulation test model, the normal forces on the four wheels are revealed as shown in Figure 5.

Note that the wild fluctuations occur when the handwheel angle rate changes rapidly, especially within the time ranges of $[1.5 \mathrm{~s}, 1.75 \mathrm{~s}]$ and $[4.25 \mathrm{~s}, 4.5 \mathrm{~s}]$. The curves in Figure 5 are roughly in mirrorimage, in which the crests of left-wheel normal force are corresponding to the troughs of the rightwheel normal force. This pattern indicates that the load on the wheels shifts from one side to another. Also, the test result showed that the minimum force over the right-front wheel comes close to only $400 \mathrm{~N}$, a sign that the vehicle is about to lift off the ground.

The roll angle and lateral acceleration of the original vehicle in response to the steering input are shown in Figure 6, respectively. As shown in Figure 6, the peak value of the roll angle is roughly 7.5 degree; meanwhile, the lateral acceleration is about $10 \mathrm{~m} / \mathrm{s}^{2}$. We will try to reduce the maximum roll angle to 5 degrees as our next goal for testing the control model.

Since the roll angle and lateral acceleration of the vehicle are already built in the simulations, we simplified the mitigation control for passive suspension, in which the changes in the spring stiffness and the damper damping coefficient of the vehicle have already been incorporated into the simulation at the beginning. Using the mitigation control described in the previous section, the adjustments can be easily determined as $\Delta k / k_{0}=0.27, \Delta c / c_{0}=0.75$.

The response of the modified prototype to the same steering input is illustrated in Figure 7, which shows that the transient state of the roll angle is much smoother and the maximum steady value is controlled below the desired degree.

LTR is another important index used to evaluate vehicle status, and it ranges from -1 to 1 . As indicated by R.C.Lin, when LTR equals -1 or 1 either the left or the right side tires lift off the ground and a smaller value stands for a more stable vehicle [16]. Figure 8 shows the LTRs of both the modified and original vehicles, and it is evident that the modified vehicle features a smaller LTR than the original one does. Moreover, the normal forces over the four wheels are also improved as shown in Figure 9.

In summary, simulation results strongly supported the proposed mitigation control, of which the contribution to vehicle stabilization is evident.

\section{Conclusion}

Vehicle rollover is a complex process. To investigate the vehicle transient and steady states, an improved rollover model was established in this study. Based on the model, we further develop a method to limit the overshoot by adjusting the relationship between the spring stiffness and the damper damping coefficient. Additional findings include the mitigation control on vehicle stabilization, and the control logic was verified by a series of simulation tests using a midsize SUV model. Simulation results indicated a decreased overshoot of the roll angle and a better confined steady value. The adjusting method proposed in this paper may help the design of both passive and active suspension controls to increase vehicle stability. We are confident that, with additional studies, the proposed model will be applicable for a real vehicle in the near future.

\section{Acknowledgments}

This project is supported by the Innovation Team Program at Science and Technology Department of Zhejiang Province in 


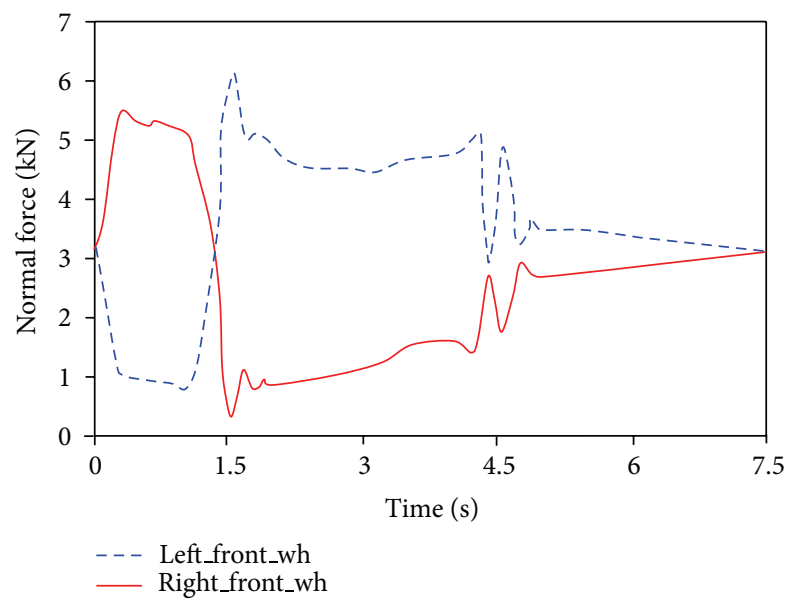

(a)

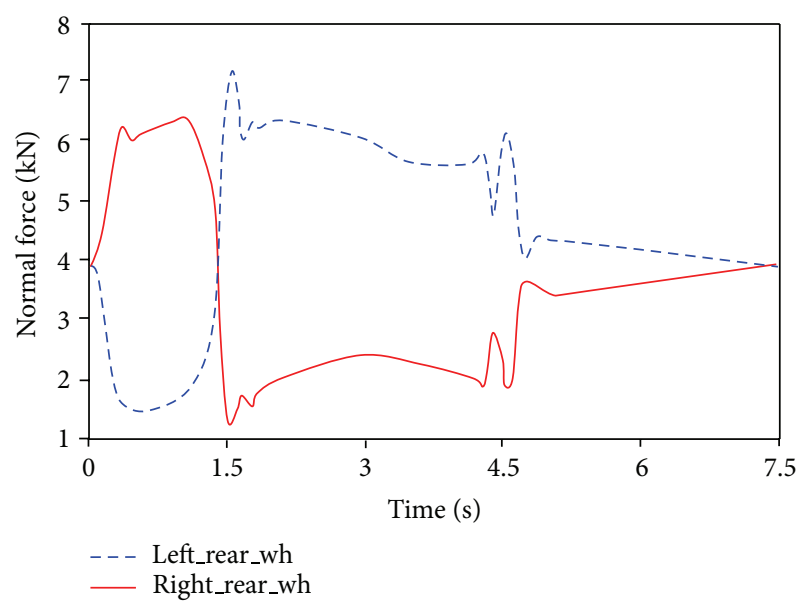

(b)

Figure 9: Normal forces on four wheels of the modified vehicle.

China (no. 2010R50045), the National Natural Science Foundation of China (no. 61074143), and the China Postdoctoral Science Foundation (no. 20100481408).

\section{References}

[1] National Highway Traffic Safety Administration, "An analysis of motor vehicle rollover crashes and injury outcomes," DOT HS $810741,2007$.

[2] A. Hac, "Rollover stability index including effects of suspension design," SAE Paper 2002-01-0965, 2002.

[3] N. Bouton, R. Lenain, B. Thuilot, and J. C. Fauroux, "A rollover indicator based on the prediction of the load transfer in presence of sliding: application to an all terrain vehicle," in Proceedings of the IEEE International Conference on Robotics and Automation (ICRA '07), pp. 1158-1163, Roma, Italy, April 2007.

[4] K. Yi, J. Yoon, and D. Kim, "Model-based estimation of vehicle roll state for detection of impending vehicle rollover," in Proceedings of the American Control Conference (ACC '07), pp. 1624-1629, New York, NY, USA, July 2007.

[5] Seongjin Yim, "Design of a preview controller for vehicle rollover prevention," IEEE Transactions on Vehicular Technology, vol. 60, no. 9, pp. 4217-4226, 2011.

[6] R. Mathieu, L. Roland, T. Benoit, and D. Christophe, "Online estimation of a stability metric including grip conditions and slope: application to rollover prevention for All-Terrain Vehicles," in Proceedings of the IEEE International Conference on Intelligent Robots and Systems (IROS '11), pp. 4569-4574, 2011.

[7] B.-C. Chen and H. Peng, "Differential-braking-based rollover prevention for sport utility vehicles with human-in-the-loop evaluations," Vehicle System Dynamics, vol. 36, no. 4-5, pp. 359389, 2001.

[8] S. B. Lu, S. B. Choi, Y. N. Li, M. S. Seong, and J. S. Han, "Global integrated control of vehicle suspension and chassis key subsystems," Proceedings of the Institution of Mechanical Engineers D, vol. 224, no. 4, pp. 423-441, 2010.

[9] S. Solmaz, M. Corless, and R. Shorten, "A methodology for the design of robust rollover prevention controllers for automotive vehicles: part 1-differential braking," in Proceedings of the 45th
IEEE Conference on Decision and Control 2006 (CDC '06), pp. 1739-1744, San Diego, Calif, USA, December 2006.

[10] V. Cherian, R. Shenoy, and T. D. Gillespie, "Model-Based Design of a SUV anti-rollover control system," SAE Paper 2008-01-0579, 2008.

[11] V. Tsourapas, D. Piyabongkarn, A. C. Williams, and R. Rajamani, "New method of identifying real-time predictive lateral load transfer ratio for rollover prevention systems," in Proceedings of the American Control Conference (ACC '09), pp. 439-444, St. Louis, Mo, USA, June 2009.

[12] H. Peiman, "Fuzzy logic controller designing of an active roll control system for medium and large size vehicles," Applied Mechanics and Materials, vol. 110-116, pp. 4845-4855, 2012.

[13] H. Peiman, "Fuzzy logic controller designing of an active roll control system for medium and large size vehicles," in Proceedings of the 2nd International Conference on Mechanical and Aerospace Engineering (ICMAE '11), July 2011.

[14] J. Garrick Forkenbrock, W. Riley Garrott, M. Mark Heitz, and B. C. O'Harra, "An experimental examination of J-Turn and Fishhook manenvers that may induce on-road, untripped, light vehicle rollover," SAE Paper 2003-01-1008, 2003.

[15] R. C. Lin, D. Cebon, and D. J. Cole, "Active roll control of articulated vehicles," Vehicle System Dynamics, vol. 26, no. 1, pp. 17-43, 1996.

[16] D. Thomas Gillespie, Fundamentals of Vehicle Dynamics, SAE International, 1992. 


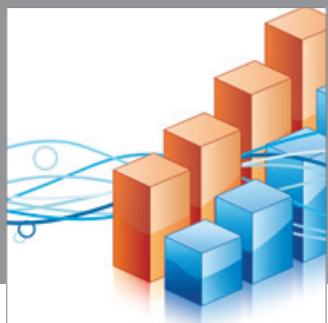

Advances in

Operations Research

mansans

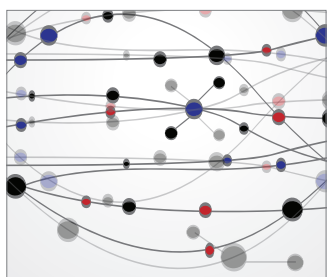

The Scientific World Journal
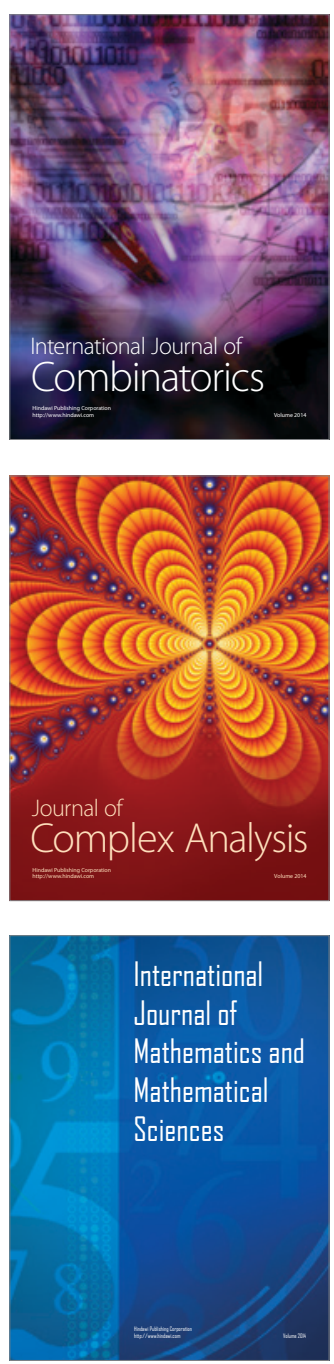
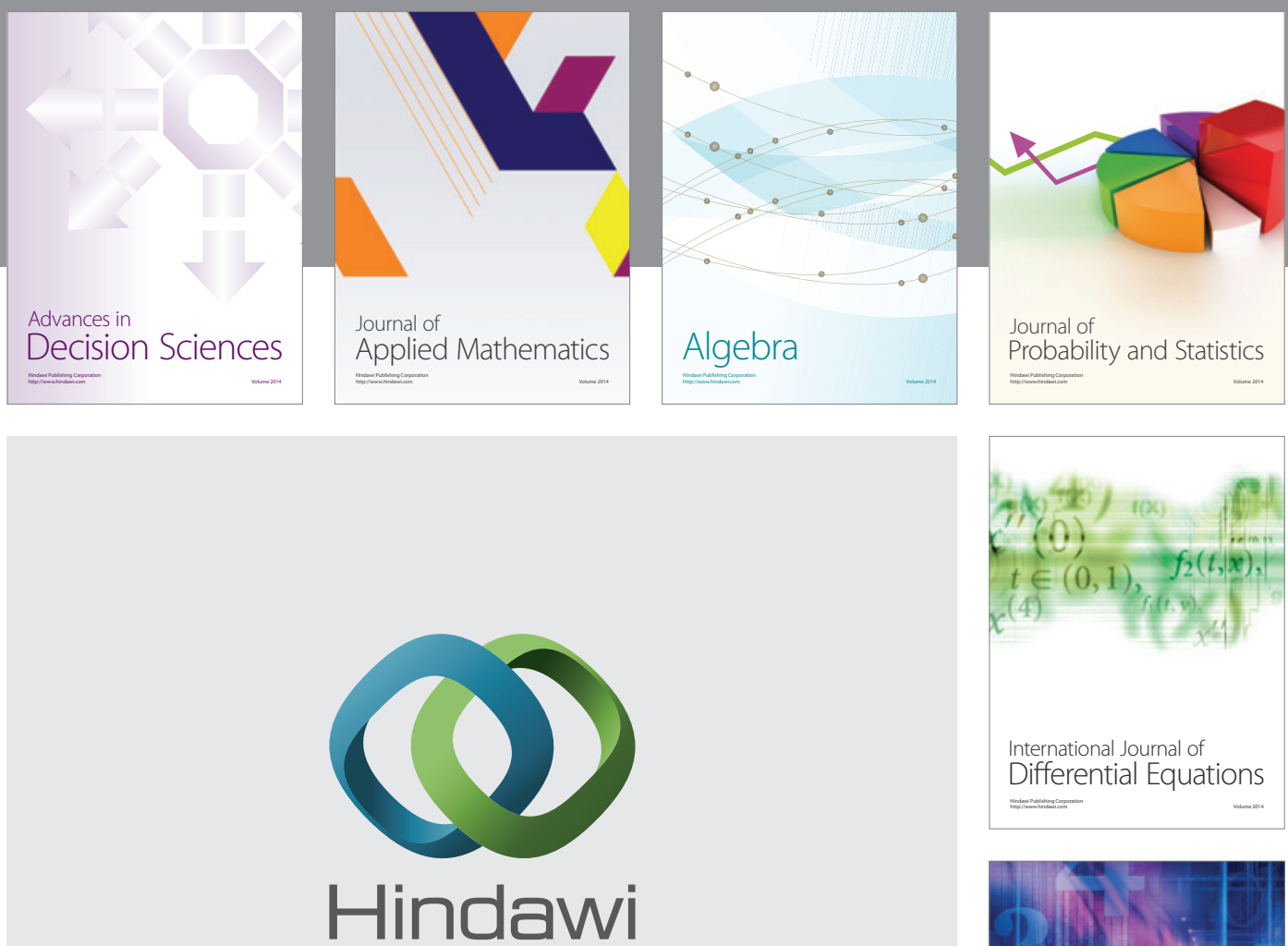

Submit your manuscripts at http://www.hindawi.com
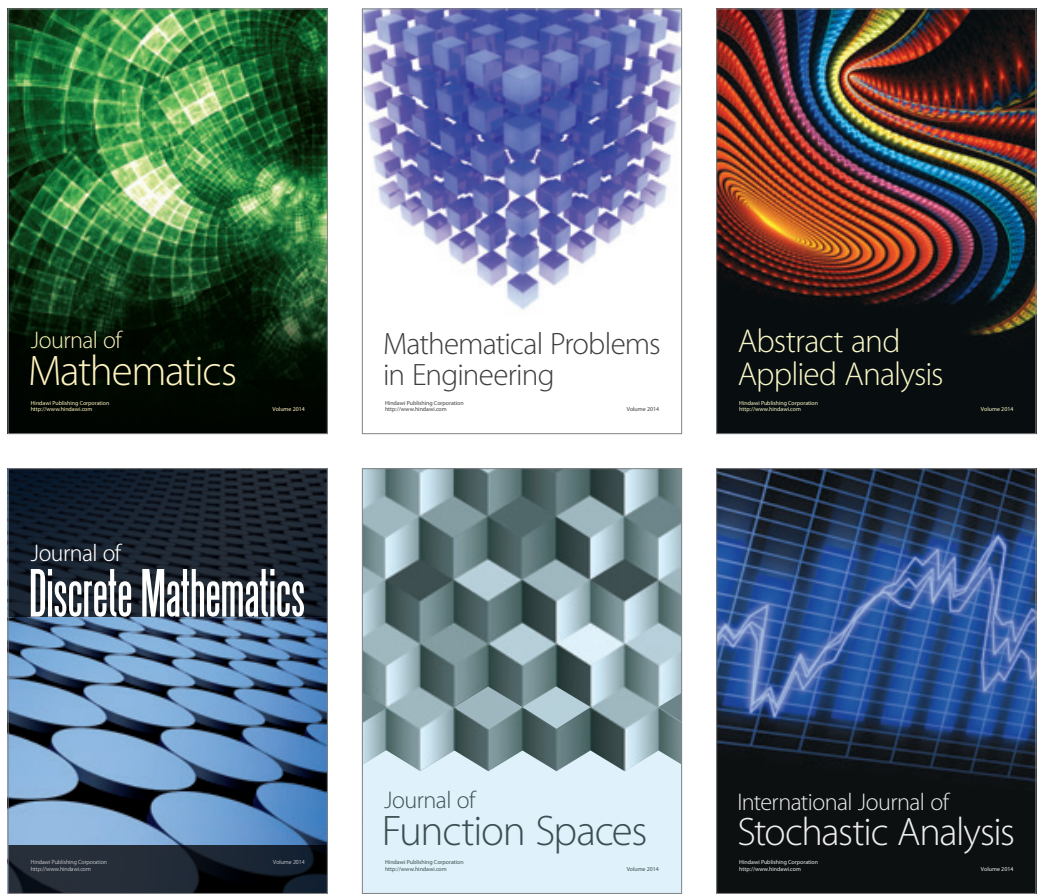

Journal of

Function Spaces

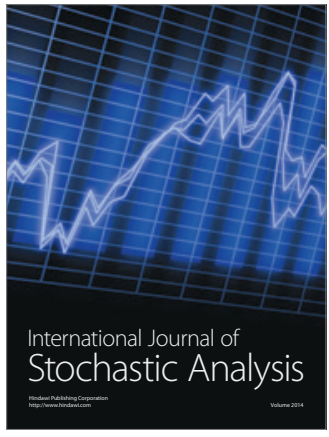

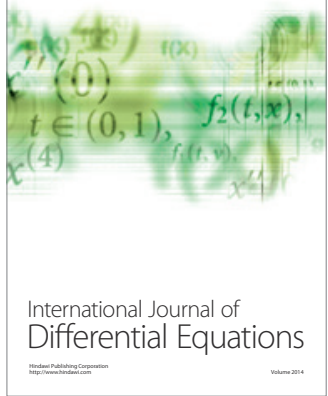
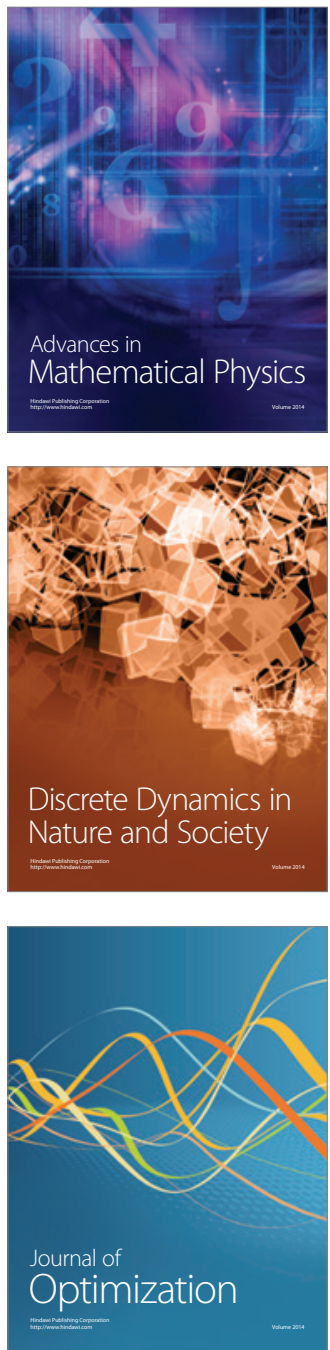